\title{
Caracterização citológica dos hemócitos de Anticarsia gemmatalis (Lepidoptera, Noctuidae) em larvas resistentes ao vírus $\mathrm{AgMNPV}$
}

\author{
Maria C. C. de Negreiro ${ }^{1}$, Renata B. R. Carvalho ${ }^{1}$, Fábio G. de Andrade ${ }^{1}$, Sheila M. Levy ${ }^{1}$, \\ Flávio Moscardi ${ }^{2} \&$ Ângela M. F. Falleiros ${ }^{1}$
}

1. Departamento de Histologia, Centro de Ciências Biológicas, Universidade Estadual de Londrina, Rodovia Celso Garcia Cid Km 380 , Caixa Postal 6001, 86051-990 Londrina, PR, Brasil. (angefal@uel.br)

2. EMBRAPA Soja, Rodovia Carlos João Strass - Distrito de Warta, Caixa Postal 231, 86001-970 Londrina, PR, Brasil. (moscardi@cnpso.embrapa.br)

\begin{abstract}
Citological characterization of the Anticarsia gemmatalis (Lepidoptera, Noctuidae) hemocytes in resistant larvae to the virus AgMNPV. The occurrence of Anticarsia gemmatalis (Hübner, 1932) larvae resistant to the virus AgMNPV in laboratory led to the study of the hemocytes of this insect, in order to evalue its participation in the mechanisms which enables the resistance to the virus. The resistant larvae with 6 to 11 developmental days old ( $3^{\text {rd }}$ to $5^{\text {th }}$ instars) were anesthetized by cold and after that were quickly cleaned in $70 \%$ alcohol. The hemolymph was collected through abdominal puncturing; the morphological analysis was made in phase contrast and colored smears with Seller solution. The total hemocytes counting (THC), was made in modified Neubauer chamber with not diluted hemolymph. For the differential hemocytes counting (DHC), was used diluted hemolymph in anticoagulant solution for insects. Six hemocytes types were identified: plasmatocytes $(38.5 \%)$, granulocytes $(22.6 \%)$, oenocytoids $(20.4 \%)$, spherulocytes $(14.5 \%)$, prohemocytes $(2.3 \%)$ and vermiform $(1.5 \%)$. The total number of hemocytes showed a significant increase during the studied larval period.
\end{abstract}

KEYWORDS. Insecta, velvetbean caterpillar, hemolymph, morphology, hemocytes counting.

RESUMO. A ocorrência de larvas de Anticarsia gemmatalis (Hübner, 1932) resistentes ao vírus AgMNPV em laboratório levou ao estudo dos hemócitos deste inseto para avaliar sua participação nos mecanismos que possibilitam a resistência ao vírus. As larvas resistentes com 6 - 11 dias de desenvolvimento ( $3^{\circ}$ a $5^{\circ}$ instar) foram anestesiadas por resfriamento e rapidamente limpas em álcool $70 \%$. A hemolinfa foi coletada através de punção abdominal, a análise morfológica foi realizada em contraste de fase e esfregaços corados com solução de Seller. A contagem total de hemócitos $(\mathrm{CTH})$ foi realizada em câmara de Neubauer com hemolinfa não diluída. Para a contagem diferencial de hemócitos $(\mathrm{CDH})$, utilizou-se hemolinfa diluída em solução anticoagulante para insetos. Foram identificados seis tipos de hemócitos: plasmatócitos $(38,5 \%)$, granulócitos $(22,6 \%)$, oenocitóides $(20,4 \%)$, esferulócitos $(14,5 \%)$, prohemócitos $(2,3 \%)$ e vermiformes $(1,5 \%)$. O número total de hemócitos mostrou um aumento significativo durante o período larval estudado.

PALAVRAS-CHAVE. Insecta, lagarta da soja, hemolinfa, morfologia, quantificação de hemócitos.

A cultura da soja é de grande importância para o complexo agroindustrial do Brasil e estrategicamente os incentivos científicos e tecnológicos para aumento da qualidade e produtividade deste grão têm sido intensificados. $\mathrm{O}$ combate às pragas que atacam a soja é cada vez mais criterioso, sendo que o controle da lagarta da soja, Anticarsia gemmatalis (Hübner, 1932) é realizado preferencialmente pela utilização de um patógeno específico, o nucleopoliedrovírus multicapsídeo Baculovirus anticarsia (AgMNPV) (MosCARDI \& SoUZA, 2002).

O uso crescente desta metodologia em laboratório e sob pressão de seleção, levou ao surgimento de larvas resistentes ao $A g \mathrm{MNPV}$, o que tem preocupado os pesquisadores e gerado estudos sobre a condição de resistência destas larvas. A preocupação é procedente, uma vez que a resistência de insetos a patógenos é similar à resistência dos mesmos aos inseticidas químicos. Além disso, o número de casos de resistência em insetos-praga cresce exponencialmente e acredita-se que a lagarta da soja também possa desenvolver alguma resistência ao seu controle viral em condições naturais de campo (Авот et al., 1996).

De modo geral, o controle biológico é efetivado com sucesso quando o patógeno consegue escapar das reações de defesa do hospedeiro. Essas reações de defesa estão intimamente relacionadas às barreiras físicas e imunológicas, sendo esta última desempenhada principalmente pelos hemócitos (RATCLIFFE \& ROWLEY, 1975; BARRACO \& MENEZES, 1985; GuPTA, 1985; RATCLIFFE $e t$ al., 1985; FALLEIROS \& GREGÓRIO, 1995; Russo et al., 2001).

Apesar do alto investimento tecnológico e do amplo conhecimento sobre a biologia deste inseto, pouco se conhece sobre os mecanismos de defesa de A. gemmatalis relacionados aos hemócitos, sendo raros os relatos obtidos na literatura, como o de ANDRADE et al. (2003) em larvas suscetíveis ao $A g \mathrm{MNPV}$. Além disso, na literatura não se encontram dados relacionando hemócitos $\mathrm{e}$ resistência em larvas de A. gemmatalis.

Assim, este trabalho teve por objetivos caracterizar morfológica e quantitativamente os hemócitos de $A$. gemmatalis em larvas resistentes ao $A g \mathrm{MNPV}$ e comparar aos dados obtidos em estudos anteriores para larvas de A. gemmatalis suscetíveis ao $A g \mathrm{MNPV}$, no intuito de elucidar a participação destas células na resistência/ suscetibilidade do inseto ao vírus.

\section{MATERIAL E MÉTODOS}

Foram utilizadas larvas resistentes (LR) de $3^{\circ}$ a $5^{\circ}$ instar (6 a 11 dias de desenvolvimento) de A. gemmatalis 
provenientes do Laboratório de Entomologia da EMBRAPA Soja/Londrina, Paraná onde são criadas em condições ambientais climatizadas (temperatura $25-27^{\circ} \mathrm{C}$, umidade relativa, $80 \%$, fotofase, $14 \mathrm{~h}$ ) e alimentadas com dieta artificial à base protéica (HOFFMANN-CAMPO et al., 1985). Os demais procedimentos foram desenvolvidos no Laboratório de Histologia da Universidade Estadual de Londrina, Paraná.

As larvas foram anestesiadas por refrigeração $\left(0^{\circ} \mathrm{C}\right.$, 5 min) e após rápida limpeza com álcool $70 \%$, tiveram sua hemolinfa coletada através de punção abdominal, com auxílio de uma pipeta Pasteur, previamente embebida em solução anticoagulante para inseto (SAI) (LeONARD et al., 1985).

Para observação a fresco, uma gota de hemolinfa foi colocada em lâmina de vidro recoberta por lamínula e observada ao microscópio de luz com contraste de fase de campo escuro. Foram também feitos esfregaços de hemolinfa, secados ao ar, corados por 20 segundos em solução Seller e observados em microscopia de luz convencional.

A contagem total de hemócitos (CTH) foi realizada com hemolinfa não diluída, em câmara de Neubauer modificada. O delineamento experimental foi inteiramente casuístico com 15 repetições por idade larval. Para a contagem diferencial dos hemócitos (CDH) utilizou-se uma gota de hemolinfa diluída em SAI (1:2). O delineamento experimental foi também inteiramente casuístico sendo utilizadas 15 larvas, numa amostragem de 200 células/indivíduo, perfazendo 3.000 células por dia de desenvolvimento.

Os dados da CTH e da CDH foram analisados através do teste de Kruskall-Wallis, complementado por Dunn, segundo ZAR (1996), com 5\% de significância.

\section{RESULTADOS E DISCUSSÃO}

Foram identificados seis tipos de hemócitos presentes na hemolinfa de larvas resistentes de $A$. gemmatalis: plasmatócitos (PL), granulócitos (GR), esferulócitos (ES), vermiformes (VE), prohemócitos (PR) e oenocitoides (OE), em contraste de fase (Fig. 1) e em esfregaços de hemolinfa (Fig. 2).

Os plasmócitos eram células polimórficas, apresentando projeções citoplasmáticas; citoplasma abundante e homogêneo; núcleo central, arredondado ou ovalado e nucléolo evidente.

Os granulócitos apresentaram formato arredondado ou oval, tamanhos variáveis e projeções citoplasmáticas filopodiais (PF, Fig. 1). O citoplasma apresentou grânulos com dimensões variáveis, sendo em sua maioria incolores nos esfregaços e com diferentes graus de refringência nos preparados a fresco. $\mathrm{O}$ núcleo era central e arredondado.

Os oenocitoides eram células esféricas, de tamanhos variáveis, apresentando-se como o maior tipo celular. Seu citoplasma era homogêneo nas observações a fresco e apresentou pontuações fortemente coradas em azul nos esfregaços. O núcleo destas células era pequeno e excêntrico. Em contraste de fase, foi possível distinguir duas subpopulações de OE. Um grupo caracterizado por apresentar um processo de retração e escurecimento do citoplasma e (após alguns minutos de observação) escurecimento das regiões circunvizinhas às células. $\mathrm{O}$ outro grupo apresentou agregação de pontos escurecidos na periferia do citoplasma (GP, Fig. 1) e posterior extrusão do seu núcleo (EN, Fig. 1).

Os esferulócitos apresentaram formas que variaram de arredondadas a elípticas, desprovidos de projeções citoplasmáticas. Em contraste de fase, apresentaram grande quantidade de esférulas citoplasmáticas salientes e refringentes. Nos esfregaços tais esférulas apresentaram coloração variável, de róseo a azul. O núcleo era pequeno, esférico, central e muitas vezes camuflado pelas esférulas.

Os prohemócitos foram as menores células observadas, com formato arredondado e sem projeções citoplasmáticas. Seu citoplasma homogêneo e escasso formava um halo ao redor do núcleo, que era central e volumoso.

As vermiformes eram células com formato fusiforme, com superfície celular lisa, com núcleo alongado e central.

A CTH demonstrou que o número total de hemócitos por volume de hemolinfa aumenta ao longo do período larval considerado (Tab. I).

A frequência média dos tipos de hemócitos determinada pela CDH pôde ser expressa como $\mathrm{PL}>\mathrm{GR}>\mathrm{OE}>\mathrm{ES}>\mathrm{PR}>\mathrm{VE}$ (Tab. II $)$.

Dos seis tipos de hemócitos encontrados na hemolinfa de larvas de A. gemmatalis resistentes (LR) ao AgMNPV, cinco tipos (PL, GR, ES, PR e VE) apresentaram morfologia semelhante à descrita por

Tabela I. Contagem diferencial de hemócitos ( $\mathrm{CDH})$ em larvas de Anticarsia gemmatalis (Hübner, 1932) resistentes ao AgMNPV. *Médias seguidas de letras diferentes nas colunas indicam diferença significativa entre os dias em cada tipo celular, determinada pelo teste de Kruskal Wallis complementado por Dunn $(\mathrm{p}<0,05)$.

\begin{tabular}{cc}
\hline $\begin{array}{c}\text { Dias de } \\
\text { desenvolvimento }\end{array}$ & $\begin{array}{c}\text { Número de hemócitos por } \mu \mathrm{l} \\
\text { de hemolinfa }\end{array}$ \\
\hline 6 & $8776,7 \pm 3479,8 \mathrm{bc} *$ \\
7 & $6371,7 \pm 3307,2 \mathrm{c}$ \\
8 & $11916,7 \pm 3672,2 \mathrm{ab}$ \\
9 & $15393,3 \pm 5441,4 \mathrm{a}$ \\
10 & $14565,0 \pm 4653,5 \mathrm{a}$ \\
11 & $13090,0 \pm 4892,7 \mathrm{a}$ \\
\hline
\end{tabular}

Tabela II. Contagem total de hemócitos (CTH) em larvas de Anticarsia gemmatalis (Hübner, 1932) resistentes ao AgMNPV. *Médias \pm desvio padrão, seguidas de letras minúsculas diferentes nas colunas indicam diferença significativa entre os dias, determinada pelo teste de Kruskal Wallis complementado por Dunn (p<0,05) (ES, esferulócitos; GR, granulócitos; OE, oenocitoides; PL, plasmatócitos; PR, prohemócitos; VE, vermiformes).

\begin{tabular}{cllllll}
\hline \multirow{2}{*}{$\begin{array}{c}\text { Dias de } \\
\text { desenvolvimento }\end{array}$} & \multicolumn{5}{c}{ Frequência dos tipos de hemócitos (\%) } \\
\cline { 2 - 8 } & PL & GR & OE & ES & PR & VE \\
\hline 6 & $44,6 \mathrm{a}^{*}$ & $16,0 \mathrm{c}$ & $12,4 \mathrm{~cd}$ & $18,8 \mathrm{a}$ & $5,2 \mathrm{a}$ & $3,0 \mathrm{a}$ \\
7 & $45,4 \mathrm{a}$ & $31,4 \mathrm{a}$ & $7,8 \mathrm{~d}$ & $10,3 \mathrm{a}$ & $3,6 \mathrm{ab}$ & $1,4 \mathrm{ab}$ \\
8 & $35,4 \mathrm{~b}$ & $26,2 \mathrm{ab}$ & $18,4 \mathrm{bc}$ & $17,0 \mathrm{a}$ & $2,4 \mathrm{bc}$ & $0,4 \mathrm{bc}$ \\
9 & $38,8 \mathrm{ab}$ & $23,4 \mathrm{ab}$ & $23,9 \mathrm{ab}$ & $11,5 \mathrm{a}$ & $0,5 \mathrm{~d}$ & $0,1 \mathrm{c}$ \\
10 & $32,6 \mathrm{~b}$ & $20,9 \mathrm{bc}$ & $30,5 \mathrm{a}$ & $14,7 \mathrm{a}$ & $0,7 \mathrm{~d}$ & $0,6 \mathrm{bc}$ \\
11 & $34,0 \mathrm{~b}$ & $17,4 \mathrm{c}$ & $29,4 \mathrm{a}$ & $14,7 \mathrm{a}$ & $1,2 \mathrm{~cd}$ & $3,3 \mathrm{a}$ \\
Média & 38,5 & 22,6 & 20,4 & 14,5 & 2,3 & 1,5 \\
\hline
\end{tabular}




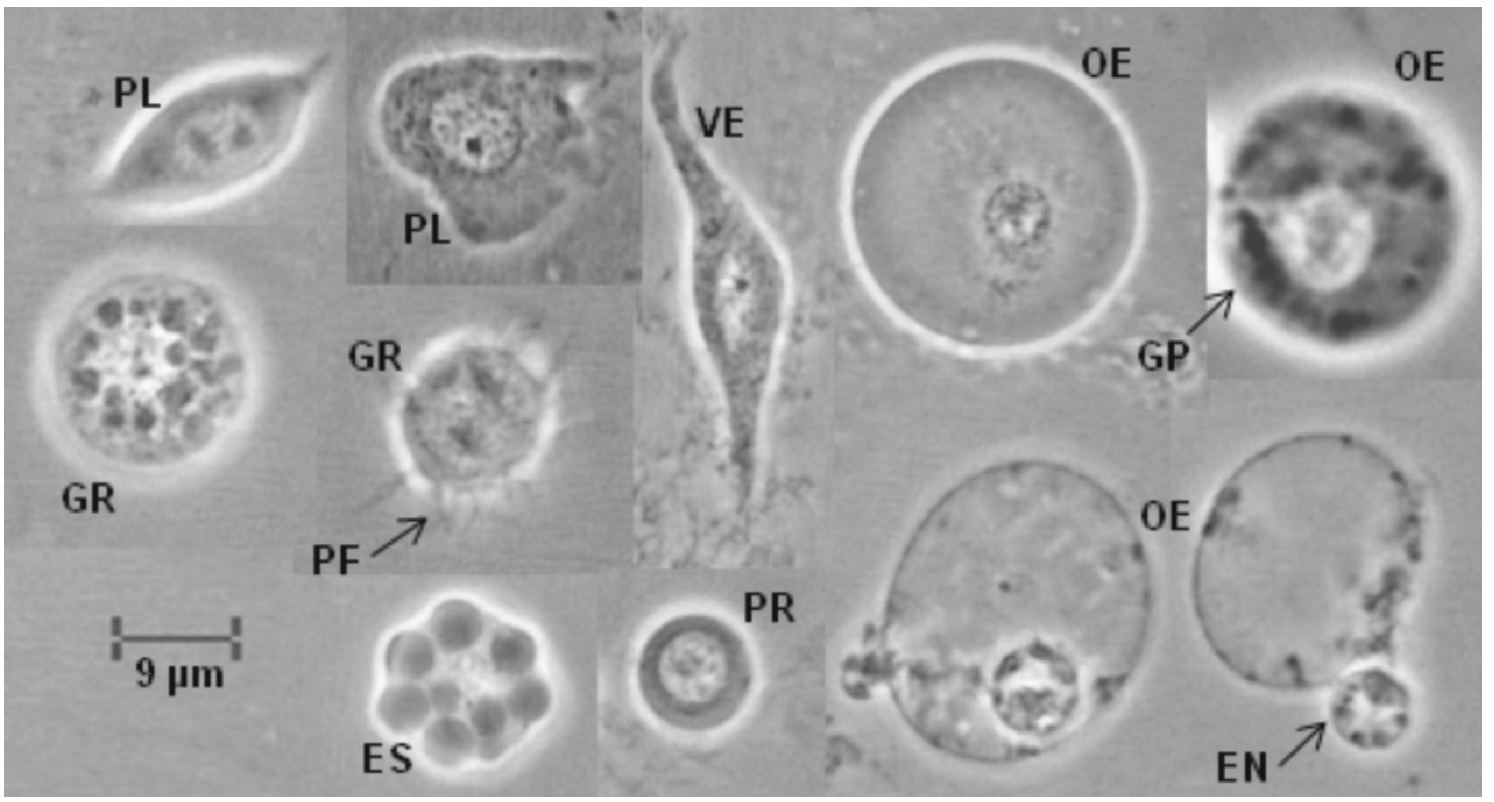

Fig. 1. Hemócitos de larvas Anticarsia gemmatalis (Hübner, 1932) resistentes ao AgMNPV. Observação a fresco em contraste de fase. (EN, extrusão do núcleo nos oenocitóides; ES, esferulócitos; GP, granulações periféricas no citoplasma dos oenocitóides; GR, granulócitos; OE, oenocitóides; PF, projeções filopodiais dos granulócitos; PL, plasmatócitos; PR, prohemócitos; VE, vermiformes).

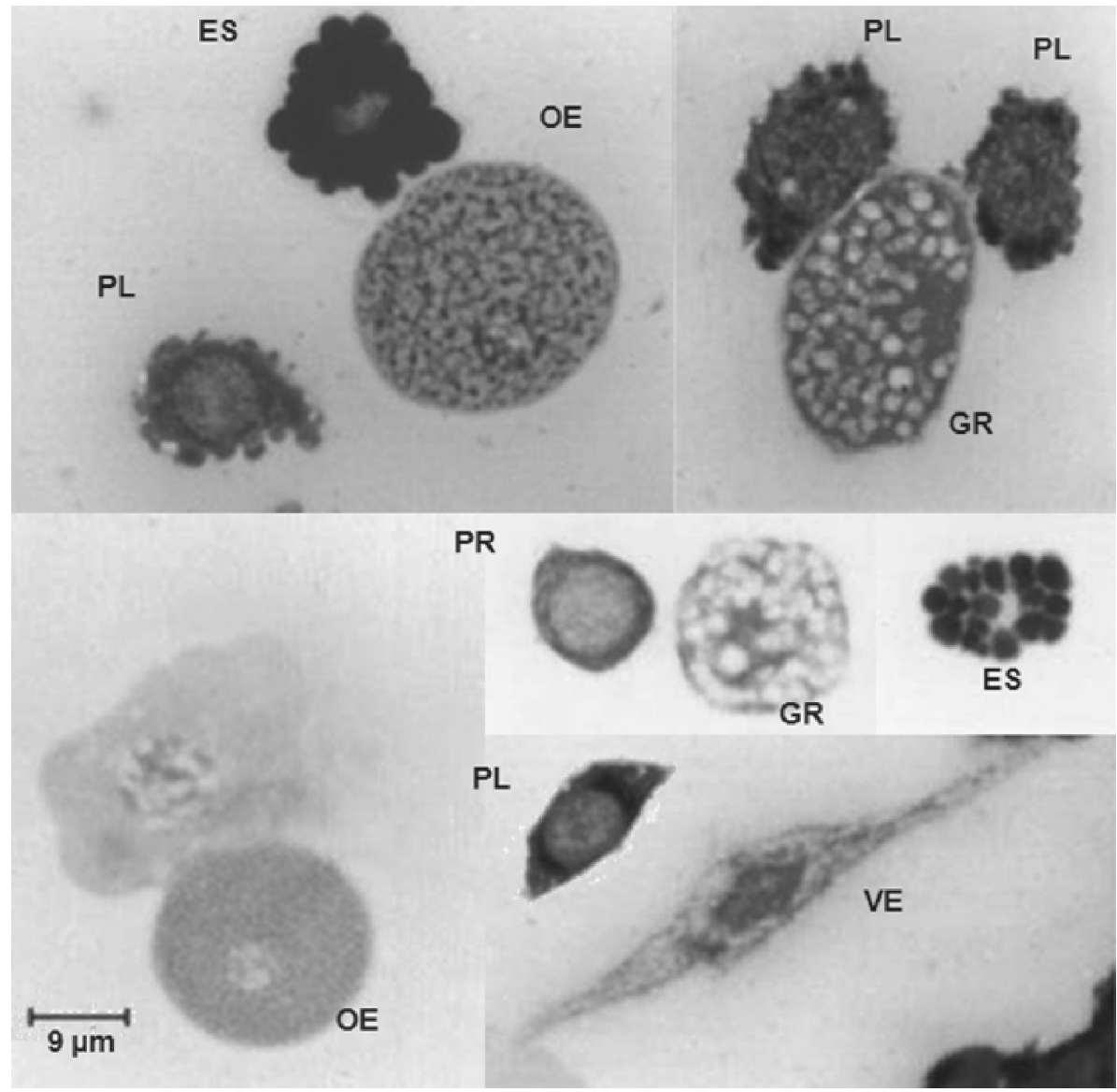

Fig. 2. Hemócitos de larvas Anticarsia gemmatalis (Hübner, 1932) resistentes ao AgMNPV. Esfregaços corados com solução de Seller (ES, esferulócitos; GR, granulócitos; OE, oenocitóides; PL, plasmatócitos; PR, prohemócitos; VE, vermiformes).

ANDRADE et al. (2003), que trabalharam com larvas suscetíveis de A. gemmatalis (LS) ao AgMNPV. Nossos resultados também estão em concordância com o descrito pela maioria dos autores para outros lepidópteros, como
AKAI \& SATO, 1976; BARDUCO et al., 1988; SAXENA et al., 1988; Geng \& DunN, 1989; BombonATO \& GREGórIo, 1995; Ribeiro et al., 1996; STRAND \& JOHNSON, 1996; RichaRdS \& EDWARDS, 1999; YAMASHITA \& IwABUCHI, 2001. 
Fato interessante foi o observado para os oenocitóides. Embora a morfologia seja a mesma relatada para LS por ANDRADE et al. (2003), foi possível verificar em LR algumas diferenças comportamentais neste tipo celular, o que nos indica a existência, nesta larva, de duas subpopulações.

Vários autores como Crossley (1979), Falleiros et al. (2003), KURIHARA et al. (1992b), LAVINE \& STRAND (2002), TANADA \& KAYA (1993) relacionaram o comportamento de retração e escurecimento citoplasmático, exibido por alguns $\mathrm{OE}$, inerente ao processo de melanização e à participação destas células no sistema de profenoloxidase (proPO). Esta enzima está diretamente relacionada com a produção de fenoloxidase, que atua no sistema de defesa do inseto contra patógenos (SöDERHÄLL, 1982). Assim, podemos inferir que as alterações exibidas pelos OE podem estar relacionadas à resistência das larvas de A. gemmatalis ao nucleopoliedrovírus $A g \mathrm{MNPV}$.

Quanto aos dados obtidos na CTH, nossos resultados em LR são discordantes dos encontrados nas LS, por ANDRADE et al. (2003), que relataram uma diminuição no número de hemócitos durante o período larval. Deste modo, a resistência deste inseto também pode ser atribuída ao maior número de hemócitos por volume de hemolinfa observado. Este fato é corroborado por autores como MosCARDi (1988) е Авот et al., (1996) que relacionam a resistência à patógenos à maior eficiência dos mecanismos de defesa nos insetos. Além disso, é sabido que a virulência do $A g \mathrm{MNPV}$ diminui ao longo do desenvolvimento larval (MosCARDI, 1988; Moscardi \& Carvalho, 1993), possivelmente contribuindo para os mecanismos de resistência.

Os resultados obtidos pela $\mathrm{CDH}$ são concordantes com as informações constantes na literatura que apontam PL e GR, como os hemócitos mais abundantes em várias espécies de insetos (Gupta, 1979, 1985; Ribeiro et al., 1996; RICHARDS \& EDWARDS, 1999; YAMASHITA \& IwABUCHI, 2001). Também foram concordantes com os encontrados por ANDRADE et al. (2003) para as LS.

Considerando a frequência média de ES, nossos resultados apontaram percentuais inferiores aos descritos para LS por ANDRADE et al. (2003), que descreveram frequência média de $20,9 \%$ para este tipo celular. À semelhança de ANDRADE et al. (2003), outros autores (Arnold \& Hinks, 1976; Barracco \& Menezes, 1985; BARDUCO et al., 1988; DRIF \& BREHÉLIN, 1993; BOMBONATO \& GREGóRIO, 1995; EdwARDS, 2004) também citam os ES como o terceiro tipo celular mais frequente nos insetos por eles estudados.

Esta frequência encontrada para os ES poderia ser explicada pelo aumento significativo de OE, sugerindo uma possível compensação entre estes tipos de hemócitos. Entretanto na literatura não existem informações sobre tal mecanismo de compensação.

Os OE em larvas resistentes apresentaram frequência superior, sendo o terceiro tipo mais encontrado. Esta frequência também é alta quando comparada à de outros lepidópteros: Bombyx mori Linnaeus, 1758 (NitTono, 1960), Hyalophora cecropia Linnaeus, 1758 (LEA \& GILBERT, 1966), Euxoa declarata Walker, 1865 (ARnold \& Hinks, 1976), Pieris rapae crucivora
Linnaeus, 1758 (TAKAda \& KitTANo, 1971), Spodoptera litura Fabricius, 1775 (KURIHARA et al., 1992a), Lacanobia oleracea Linnaeus, 1758 (RICHARDS \& EDWARDS, 1999), Diatraea saccharalis Fabricius, 1794 (BARDUCO et al., 1988; Bombonato \& Gregório, 1995; FAlleiros et al. 2003), onde este tipo celular apresentou valores médios inferiores a $10 \%$.

A frequência de OE em LR foi também superior ao encontrado por ANDRADE et al. (2003) em LS. Assim, podemos sugerir que os OE contribuem para o desenvolvimento da resistência das LR. Entretanto, como na literatura não existem relatos correlacionando este tipo celular com a resistência a patógenos, outros estudos deverão ser conduzidos visando elucidar sua efetiva participação nos mecanismos de defesa do inseto à infecção viral.

Os outros tipos celulares descritos e quantificados com baixa frequência (PR e VE, respectivamente) também são citados como células pouco abundantes nos insetos. Os resultados apresentados por estes hemócitos em LR estão em concordância com o descrito por inúmeros autores (GuPTA, 1979, 1985; GuPTA \& SuTHERLAND, 1966; Kurihara et al., 1992a; Ahmad, 1993; Bombonato \& GregóRIO, 1995; YAMASHITA \& IwABUCHI, 2001; FALLEIROS et al., 2003) para outras espécies, bem como o descrito para LS de A. gemmatalis por ANDRADE et al. (2003).

A caracterização citológica da maioria dos hemócitos das LR de A. gemmatalis, mostrou-se semelhante à das LS, com exceção dos OE que apresentaram características morfológicas e quantitativas diferenciadas nas LR, permitindo inferir que este tipo celular está envolvido na resistência deste inseto ao vírus. Além disso, pode-se considerar que o elevado número de hemócitos por volume de hemolinfa também contribui para esta resistência.

Agradecimentos. Ao $\mathrm{CNPq}$ pelo suporte financeiro, à EMBRAPA Soja pelo fornecimento das larvas de A. gemmatalis e da dieta artificial, à Inês Cristina B. da Fonseca pelo auxílio nas análises estatísticas e à Danielle B. Rissi pelo apoio técnico.

\section{REFERÊNCIAS BIBLIOGRÁFICAS}

Aвot, A. R.; Moscardi, F.; Fuxa, J. R.; Sosa-Gómez, D. R. \& Richter, A. R. 1996. Development of resistance by Anticarsia gemmatalis from Brazil and the United States to a nuclear polyhedrosis virus under laboratory selection pressure. Biological Control 9:126-130.

Ahmad, A. 1993. Effect of â-ecdsone ingestion on total and differential haemocyte counts (THC \& DHC) in tobacco caterpillar, Spodoptera litura Fabr. (Lepidoptera: Noctuidae). Bulletin de la Societe Entomologique de France 66:113-122.

AкаI, H. \& SAто, S. 1976. Surface ultraestructure of the larval hemocytes of the silkworm, Bombyx mori L. (Lepidoptera: Bombycidae). International Journal of Insect Morphology \& Embryology 5(1):17-21.

Andrade, F. G.; Negreiro, M. C. C. de; Gregório, E. A.; Moscardi, F \& Falleiros, Â. M. F. 2003. Hemocytes of Anticarsia gemmatalis (Hübner) (Lepidoptera: Noctuidae) larvae: morphological and quantitative studies. Acta Microscopica 12(1):59-64

Arnold, J. W. \& Hinks, H. 1976. Haemopoiesis in Lepidoptera. I. The multiplication of circulating haemocytes. Canadian Journal of Zoology 54:1003-1012.

Barduco, M. C.; Gregório, E. A. \& Toledo, L. A. 1988. Hemócitos de Diatraea saccharalis (Lepidoptera: Pyralidae) no período larval. Estudo morfológico e quantitativo. Revista Brasileira de Biologia 48(4):925-932. 
Barracco, M. A. \& Menezes, H. 1985. Mecanismos celulares de defesa em insetos. Ciência e Cultura 37(2):237-250.

Bombonato, M. T. S. \& Gregório, E. A. 1995. Estudo morfológico e quantitativo dos hemócitos em larvas de Diatraea saccharalis (Fabricius) (Lepidoptera, Pyralidae). Revista Brasileira de Zoologia 12(4):867-879.

Crossley, A. C. S. 1979. Biochemical and ultrastructural aspects of synthesis, storage, and secretion in hemocytes. In: GuPTA, A. P. ed. Insect hemocytes: development, forms, function and techniques. Cambridge, Cambridge University. p.423-473.

DrIF, L. \& BrÉHELIN, M. 1993. Structure, classification and functions of insect haemocytes. In: РАтатнак, J. P. N. ed. Insect Immunity. New Deli, Kluwer Academic. p.1-14

Edwards, J. P. 2004. Insect Immune Suppression: Optimisation of biological control by suppression of insect immune defences. Disponível em: <http://www.csl.gov.uk/science/organ/environ/ invertebrate/maff1.cfm>. Acesso em: 20.01.2004

Falleiros, Â. M. F. \& Gregório, E. A. 1995. Hemócitos fagocitários em larvas de Diatraea saccharalis (Fabricius) (Lepidoptera, Pyralidae). Revista Brasileira de Zoologia 12(4):751-758.

Falleiros, Â. M. F.; Bombonato, M. T. S. \& Gregório, E. A. 2003. Ultrastructural and quantitative studies of hemocytes in the sugarcane borer Diatraea saccharalis (Fabricius) (Lepidoptera: Pyralidae). Brazilian Archives of Biology and Technology 46(2):287-294.

Geng, C. \& Dunn, P. E. 1989. Plasmatocyte depletion in larvae of Manduca sexta following injection of bacteria. Developmental and Comparative Immunology 13:17-23.

Gupta, A. P. 1979. Hemocyte types: their structures, synonymies, interrelationships, and taxonomic significance. In: GuPTA, A. P. ed. Insect Hemocytes: development, forms, functions and techniques. Cambridge, Cambridge University. p.85-127. 1985. Cellular elements in the hemolymph. In: Kerkut, G. A. \& Gilbert, L. I. eds. Comprehensive Insect Physiology, Biochemistry and Pharmacology. v.3. Oxford, Pergamon. p.401-451.

Gupta, A. P. \& Sutherland, D. J. 1966. In vitro transformation of the insect plasmatocyte in some insects. Journal of Insect Physiology 12:1369-1375.

Hoffmann-CAmpo, C. B.; Oliveira, E. B. DE \& Moscardi, F. 1985. Criação massal da lagarta da soja. Londrina, EMBRAPA. 23p.

Kurihara, Y.; Shimazu, T. \& Wago, H. 1992a. Classification of hemocytes in the common cutworm, Spodoptera litura (Lepidoptera: Noctuidae): I-phase microscopic study. Applied Entomology and Zoology 27:225-235.

1992b. Classification of hemocytes in the common cutworm, Spodoptera litura (Lepidoptera: Noctuidae): II-possible roles of granular plasmatocytes and oenocytoids in the cellular defense reactions. Applied Entomology and Zoology 27:237-242.

Lavine, M. D. \& Strand, M. R. 2002. Insect hemocytes and their role in immunity. Insect Biochemistry and Molecular Biology 32:1295-1309.

LeA, M. S. \& Gilbert, L. I. 1966. The haemocytes of Hyalophora cecropia (Lepidoptera). Journal of Morphology 118:197216
Leonard, C.; Söderhäll, K. \& Ratcliffe, N. A. 1985. Studies on prophenoloxidase and protease activity of Blaberus craniifer haemocytes. Insect Biochemical 15(6):803-810.

Moscardi, F. 1988. Utilização de vírus entomopatogênicos em campo. In: Alves, S. B. ed. Controle Microbiano de Insetos. 2.ed. Piracicaba, FEALQ. p.509-539.

Moscardi, F. \& Carvalho, R. C. Z. 1993. Consumo e utilização de soja por Anticarsia gemmatalis Hüb. (Lepidoptera: Noctuidae) infectada, em diferentes estádios larvais, por seu vírus de poliedrose nuclear. Anais da Sociedade Entomológica do Brasil 22:267-280.

Moscardi, F. \& SouzA, M. L. 2002. Baculovírus para o controle de pragas. Biotecnologia Ciência e Desenvolvimento 24:22-29.

NitTono, Y. 1960. Studies on the blood cells in the silkworm Bombyx mori L. Bulletin of the Sericultural Experiment Station 16:171-266.

Ratcliffe, N. A. \& Rowley, A. F. 1975. Cellular defence reations of insect hemocytes in vitro: phagocytosis in a new suspension culture system. Journal of Invertebrate Pathology 26:225-233.

Ratcliffe, N. A.; Rowley, A. F.; Fitzgeald, S. W. \& Rhodes, C. P. 1985. Invertebrate immunity: basic concepts and recent advances. International Review of Cytology 97:183-279.

Ribeiro, C.; Simões, N. \& BrehÉLin, M. 1996. Insect immunity: the haemocytes of armyworm Mythimna unipuncta (Lepidoptera: Noctuidae) and their role defence reactions in vivo and in vitro studies. Journal of Insect Physiology 42(9):815-822.

Richards, E. H. \& Edwards, J. P. 1999. Parasitization of Lacanobia oleracea (Lepidoptera: Noctuidae) by the ectoparasitic wasp, Eulophus pernicornis: effects of parasitization, venom and starvation on host haemocytes. Journal of Insect Physiology 45: 1073-1083.

Russo, J.; BrehéLin, M. \& CARton, Y. 2001. Haemocyte changes in resistant and susceptible strains of Drosophila melanogaster caused by virulent and avirulent strains of the parasitic wasp Leptopilina boulardi. Journal of Insect Physiology 47:167-172.

Saxena, B. P.; Sharma, P. R. \& Tikku, K. 1988. Scanning eletron microscopical studies of the haemocytes of Spodoptera litura Fabr. Cytologia 53:385-391.

SöDERHÄLl, K. 1982. The prophenoloxidase activating system and melanization a recognition mecanism of atrhopods: a review. Development Comparative Immunology 6:601-611.

Strand, M. R. \& Johnson, J. A. 1996. Characterization of monoclonal antibodies to hemocytes of Pseudoplusia includens. Journal of Insect Physiology 42(1):21-31.

Takada, M. \& Kittano, H. 1971. Studies on the larval hemocytes in the cabbage with butterfly, Pieris rapae crucivora (Boisduval), with special reference to hemocyte classifications, phagocytic activity and encapsulative capacity. Kontyú 39:385-394

Tanada, Y. \& KaYA, H. K. 1993. Insect pathology. San Diego, Academic. 666p.

Yamashita, M. \& Iwabuchi, K. 2001. Bombyx mori prohemocyte division and differentiation in individual microcultures. Journal of Insect Physiology 47:325-331.

ZAR, J. H. 1996. Biostatistical analysis. $4^{\mathrm{a} e d .}$ New Jersey, Printice Hall. 663p. 